Supporting Information

for

\title{
Characterizing interfacial structures of dye-sensitized solar cell working electrodes
}

Jacqueline M. Cole ${ }^{1,2,{ }^{*}}$ and Ulrich F. J. Mayer ${ }^{1}$

1. Cavendish Laboratory, Department of Physics, University of Cambridge, J. J. Thomson Avenue, Cambridge CB3 OHE, United Kingdom

2. ISIS Neutron and Muon Source, STFC Rutherford Appleton Laboratory, Harwell Science and Innovation Campus, Didcot OX11 0QX, United Kingdom

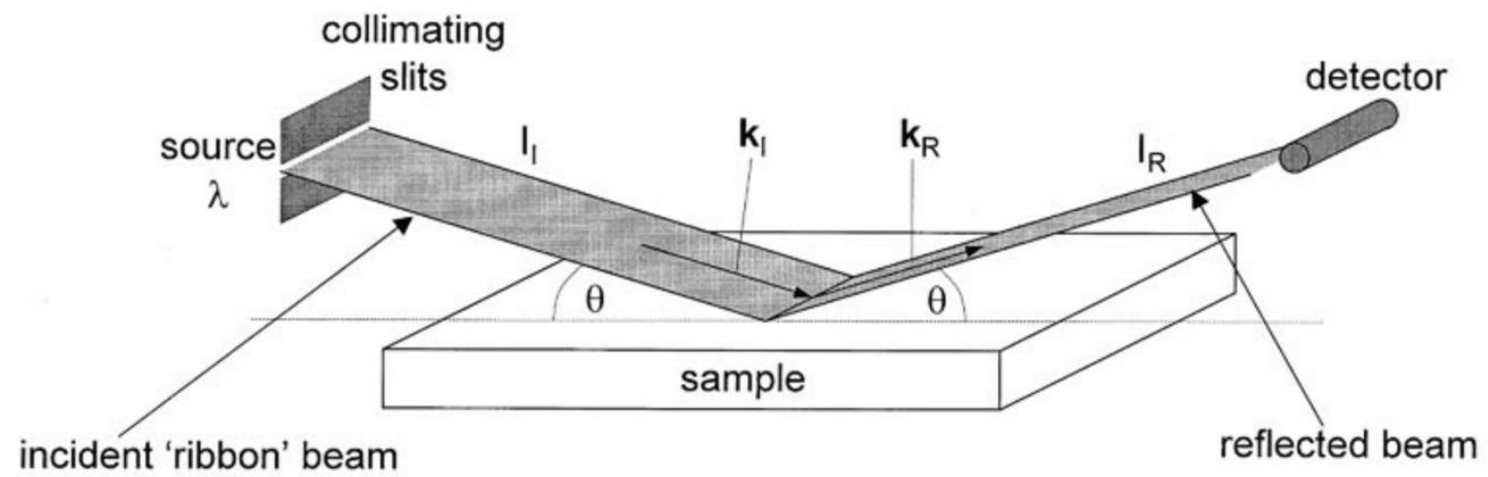

Figure S1. Schematic illustration of a reflectivity experiment (the incident angle is

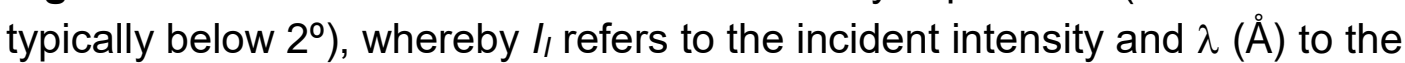
wavelength of the incident radiation beam. $I_{R}$ refers to the intensity of the reflected specular beam, while the wavevectors $k_{l}$ and $k_{R}$ define the incident and reflected beam, respectively. Reproduced with permission from ref. S1. Copyright 1999 Elsevier.

Table S1. Refined structural parameters for thin films of $\mathrm{TiO}_{2}$ sensitized with $\mathrm{N} 3$ using EtOH, EtOH:t-BuOH (1:1, v/v), DMSO, or MeCN:t-BuOH (1:1, v/v). Adapted with permission from ref. S2. Copyright 2018 American Chemical Society.

\begin{tabular}{lllll}
\hline N3 layer & EtOH & $\begin{array}{l}\text { EtOH:t-BuOH } \\
(1: 1, \mathrm{v} / \mathrm{v})\end{array}$ & DMSO & $\begin{array}{l}\text { MeCN:t-BuOH } \\
(1: 1, \mathrm{v} / \mathrm{v})\end{array}$ \\
\hline Thickness, $d_{\text {obs }}(\AA)$ & $12.3 \pm 1.1$ & $10.5 \pm 0.4$ & $6.3 \pm 0.3$ & $10.2 \pm 0.3$ \\
SLD $\left(\times 10^{-6} \AA^{-2}\right)$ & $10.5 \pm 0.2$ & $11.9 \pm 0.9$ & $12.0 \pm 0.8$ & $11.6 \pm 1.5$ \\
Surface roughness $(\AA)$ & $3.3 \pm 0.1$ & $3.8 \pm 0.3$ & $3.7 \pm 0.4$ & $3.3 \pm 0.5$ \\
\hline
\end{tabular}


Table S2. Refined structural parameters for XRR data on thin films of $\mathrm{TiO}_{2}$ sensitized with MK-2 or MK-44. Reproduced with permission from ref. S3. Copyright 2017 Royal Society of Chemistry.

\begin{tabular}{lll}
\hline Dye layer & MK-2 & MK-44 \\
\hline Thickness, $d_{\text {obs }}(\AA)$ & $23.2 \pm 0.2$ & $9.6 \pm 0.2$ \\
$S L D\left(\times 10^{-6} \AA^{-2}\right)$ & $10.1 \pm 0.4$ & $9.6 \pm 0.3$ \\
Surface roughness $(\AA)$ & $6.4 \pm 0.2$ & $3.6 \pm 0.1$ \\
\hline
\end{tabular}

Table S3. Thickness, density, SLD, and dye volume fraction values for thin films of $\mathrm{TiO}_{2}$ sensitized with solutions of MK-2, N3, or N749 of different concentration. Reproduced with permission from ref. S4. Copyright 2018 American Chemical Society.

\begin{tabular}{lllllll}
\hline & 0.50 & 1.00 & 0.50 & 1.00 & 0.50 & 1.00 \\
& \multicolumn{2}{c}{$C_{\text {MK-2 }}(\mathrm{mM})$} & \multicolumn{2}{c}{$C_{\mathrm{N} 3}(\mathrm{mM})$} & \multicolumn{2}{c}{ CN749 $^{(\mathrm{mM})}$} \\
\hline Thickness, $d_{\text {obs }}(\AA)$ & 24.16 & 38.12 & 16.09 & 20.10 & 15.23 & 16.03 \\
Density $\left(\times 10^{-4} \text { unit/ } / \AA^{3}\right)^{a}$ & 6.85 & 4.24 & 12.05 & 4.87 & 3.70 & 5.95 \\
$S L D\left(\times 10^{-6} \AA^{-2}\right)$ & 9.93 & 6.15 & 12.70 & 5.13 & 6.27 & 10.06 \\
\hline
\end{tabular}

aThe unit is defined as a dye molecule.

Table S4. AFM and XRR parameters for dye layer on thin films of $\mathrm{TiO}_{2}$ singly sensitized with 15 and co-sensitized with XS6 and 15; reproduced with permission from ref. S5. Copyright 2018 Wiley.

\begin{tabular}{|c|c|c|c|c|c|c|c|c|}
\hline \multirow[b]{2}{*}{ Sample } & \multicolumn{4}{|c|}{ AFM parameters } & \multicolumn{4}{|c|}{ XRR parameters } \\
\hline & $\begin{array}{l}\text { Mean } \\
\text { hight } \\
{[\mathrm{nm}]}\end{array}$ & $\begin{array}{l}\text { Max } \\
\text { hight } \\
{[\mathrm{nm}]}\end{array}$ & $\begin{array}{l}\text { Aggregate } \\
\text { coverage } \\
{[\%]}\end{array}$ & $\begin{array}{l}\text { Number of } \\
\text { aggregates } \\
{\left[\mu \mathrm{m}^{-2}\right]}\end{array}$ & $\begin{array}{l}\text { Dye-layer } \\
\text { thickness, } \\
\left.d_{\text {obs }}[\AA]\right]\end{array}$ & $\begin{array}{l}\text { SLD dye }_{\text {dx }}[\times \\
\left.10^{-6} \AA^{-2}\right]\end{array}$ & $\begin{array}{l}\text { Surface } \\
\text { roughness } \\
{[\AA]}\end{array}$ & $\begin{array}{l}\text { Surface } \\
\text { coverage } \\
{[\%]}\end{array}$ \\
\hline 15 & $8 \pm 2$ & $15 \pm 3$ & $7 \pm 2$ & $1.1 \pm 0.4$ & $24.3 \pm 0.3$ & $7.8 \pm 0.4$ & $2.7 \pm 0.3$ & $62 \pm 3$ \\
\hline XS6 then 15 & $6 \pm 1$ & $8 \pm 2$ & $0.7 \pm 0.3$ & $0.8 \pm 0.3$ & $18.8 \pm 0.3$ & $8.7 \pm 0.5$ & $3.6 \pm 0.4$ & $72 \pm 4$ \\
\hline XS6 and 15 & $7.8 \pm 0.7$ & $11 \pm 1$ & $0.3 \pm 0.1$ & $0.24 \pm 0.09$ & $18.6 \pm 0.3$ & $8.8 \pm 0.5$ & $3.4 \pm 0.4$ & $73 \pm 4$ \\
\hline
\end{tabular}

Table S5. Structural parameters determined by in-situ NR for $\mathrm{TiO}_{2}$ substrates sensitized with MK-2 or MK-44 dyes, submerged in a solution of $\mathrm{d}_{3}-\mathrm{MeCN}, 0.7 \mathrm{M}$ Lil in $\mathrm{d}_{3}-\mathrm{MeCN}$, or $0.7 \mathrm{M} \mathrm{Lil}$ and $0.05 \mathrm{M} \mathrm{I}_{2}$ in $\mathrm{d}_{3}-\mathrm{MeCN}$. Reproduced with permission from ref. S3. Copyright Royal Society of Chemistry.

\begin{tabular}{|c|c|c|c|}
\hline \multirow[b]{2}{*}{ Dye } & \multirow[b]{2}{*}{ Solution } & \multicolumn{2}{|l|}{ Dye } \\
\hline & & $\begin{array}{l}\text { Thickness, } d_{o b s} / \\
\AA\end{array}$ & $\operatorname{SLD}_{n}\left(\times 10^{-6}\right) / \AA^{-2}$ \\
\hline \multirow[t]{3}{*}{ MK-2 } & $\mathrm{d}_{3}-\mathrm{MeCN}$ & $23.6 \pm 1.9$ & $1.9 \pm 0.1$ \\
\hline & 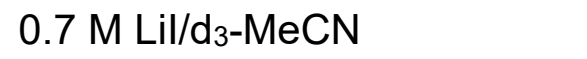 & $23.8 \pm 1.9$ & $2.5 \pm 0.1$ \\
\hline & $0.7 \mathrm{M}$ Lil / $0.05 \mathrm{M} \mathrm{I}_{2} / \mathrm{d}_{3}-\mathrm{MeCN}$ & $22.2 \pm 1.5$ & $2.4 \pm 0.1$ \\
\hline MK-44 & $\mathrm{d}_{3}-\mathrm{MeCN}$ & $9.2 \pm 0.7$ & $2.9 \pm 0.6$ \\
\hline
\end{tabular}



0.7 M Lil//d3-MeCN
$15.9 \pm 1.0$
$3.6 \pm 0.4$
$0.7 \mathrm{M} \mathrm{Lil} \mathrm{/} 0.05 \mathrm{M} \mathrm{I}_{2} / \mathrm{d}_{3}-\mathrm{MeCN}$
$15.9 \pm 1.0$
$3.8 \pm 0.4$

Table S6. Co-refined structural parameters of the dye layers (or the top layer where specified) for two contrast-matching scenarios ( $\mathrm{d}_{3}-\mathrm{MeCN}$ and Si-contrast-matched $\mathrm{d}_{3}-\mathrm{MeCN} / \mathrm{MeCN}$ ) in the presence of $\mathrm{Lil}$ or $\mathrm{Lil} / \mathrm{I}_{2}$ under consideration of the solvent ingression in the dye layer (or the top layer where specified). Reproduced with permission from ref. S6. Copyright 2021 American Chemical Society.

\begin{tabular}{|c|c|c|c|c|c|c|}
\hline \multirow[b]{2}{*}{ Dye } & \multicolumn{6}{|c|}{ Dye layer (or top layer where specified) } \\
\hline & Solution & $\begin{array}{l}\text { Thickness, } \\
d_{\text {obs }}(\AA)\end{array}$ & $\begin{array}{l}\text { Fitting error } \\
\text { for thickness } \\
\text { (Å) }\end{array}$ & $\begin{array}{l}\text { Density of the } \\
\text { dye }\left(\rho d y e \text { in } 10^{-4}\right. \\
\text { unit } / A^{3} \text { ) or top } \\
\text { layer }\left(\rho \text { top in } 10^{-4}\right. \\
\left.\text { unit }\left(I_{2}\right) / A^{3}\right)\end{array}$ & $\begin{array}{l}\text { Fitting } \\
\text { error for } \rho \\
\left(10^{-5}\right. \\
\left.\text { unit/A } A^{3}\right)\end{array}$ & $\begin{array}{l}\text { Dye coverage } \\
\varphi_{d y e} \text { or } \\
\text { electrolyte } \\
\text { coverage } \varphi_{e} \\
(\%)\end{array}$ \\
\hline \multirow[t]{4}{*}{ MK-2 } & $\mathrm{MeCN}$ & 20.6 & $-0.7,0.8$ & 5.84 & $-3.1,3.5$ & 91.4 \\
\hline & MeCN + Lil & 20.6 & $-0.6,0.8$ & 5.86 & $-3.5,2.6$ & 87.8 \\
\hline & $\begin{array}{l}\mathrm{MeCN}+\mathrm{Lil}+ \\
\mathrm{I}_{2}\end{array}$ & 20.8 & $-1.0,0.9$ & 5.97 & $-5.1,6.5$ & 62.7 \\
\hline & (top layer) & 30.3 & $-1.0,1.5$ & 0.01025 & $-8.0,6.9$ & 11.8 \\
\hline \multirow[t]{4}{*}{ N3 } & $\mathrm{MeCN}$ & 11.5 & $-0.5,0.5$ & 9.95 & $-5.8,7.3$ & 92.1 \\
\hline & MeCN + Lil & 11.6 & $-0.4,0.5$ & 9.75 & $-5.4,8.2$ & 78.8 \\
\hline & $\begin{array}{l}\mathrm{MeCN}+\mathrm{Lil}+ \\
\mathrm{I}_{2}\end{array}$ & 13.3 & $-0.3,0.3$ & 9.90 & $-2.3,2.1$ & 99.7 \\
\hline & (top layer) & 22.2 & $-0.4,0.4$ & 0.01054 & $-5.4,8.2$ & 4.5 \\
\hline \multirow[t]{4}{*}{ N749 } & $\mathrm{MeCN}$ & 10.3 & $-0.5,0.3$ & 7.63 & $-6.1,5.5$ & 55.7 \\
\hline & MeCN + Lil & 10.9 & $-0.6,0.8$ & 7.74 & $-7.7,8.0$ & 36.3 \\
\hline & $\begin{array}{l}\mathrm{MeCN}+\mathrm{Lil}+ \\
\mathrm{I}_{2}\end{array}$ & 12.5 & $-0.7,0.7$ & 12.81 & $-8.2,3.6$ & 99.9 \\
\hline & (top layer) & 35.4 & $-1.2,1.4$ & 0.01066 & $-7.6,4.1$ & 5.5 \\
\hline
\end{tabular}

aThe 'unit' density in the table is defined as the density per dye molecule or $\mathrm{I}_{2}$. The neutron-scattering length density (SLD) of each dye layer can be derived by multiplying the fitted density of each dye layer with the total SLD of one dye molecule.

Table S7. Dye-layer thickness for MK-2, N3, and N749 determined by various forms of NR and XRR in this work and in previous work carried out on the same dyes at the same sensitization concentration $(0.3 \mathrm{mM})$ and time $(20 \mathrm{~h})$ using the same solvents. Reproduced with permission from ref. S6. Copyright 2021 American Chemical Society.

\begin{tabular}{lllll}
\hline & $\begin{array}{l}\text { Dye-layer thickness, } \boldsymbol{d}_{\text {obs }} \\
(\mathbf{A})\end{array}$ & $\mathbf{N 3}$ & $\mathbf{N 7 4 9}$ & $\mathbf{M K - 2}$ \\
\hline $\begin{array}{l}\text { Work from ref. } \\
\text { S6 }\end{array}$ & & & & \\
& & & & \\
& $\begin{array}{l}\text { in situ NR } \\
\text { (INTER, triple co-refinement) } \\
\text { ex situ XRR } \\
\text { ex situ NR/XRR co- }\end{array}$ & $11.5 \pm 0.5$ & $10.3+0.3 /-0.5$ & $20.6 \pm 0.8$ \\
& refinement & $10.7 \pm 0.3$ & $11.2+0.3 /-0.1$ & $19.4+0.7 /-0.4$ \\
& & & $10.5+0.3 /-0.2$ & $19.5+1.0 /-1.3$ \\
Work from refs. & & & & \\
S2, S3, S7 & in situ NR & $11.8 \pm 1.2$ & & $23.6 \pm 1.9$ \\
& ex situ XRR & $10.2 \pm 0.3$ & & $23.2 \pm 0.2$ \\
\hline
\end{tabular}




\section{References}

S1. Lovell, M. R.; Richardson, R. M. Analysis methods in neutron and X-ray reflectometry. Curr. Opin. Colloid Interface Sci. 1999, 4, 197-204.

S2. $\quad$ Cole, J. M.; Gong, Y.; McCree-Grey, J.; Evans, P. J.; Holt, S. A. Modulation of N3 and N719 dye $\cdots \mathrm{TiO}_{2}$ Interfacial Structures in DyeSensitized Solar Cells As Influenced by Dye Counter Ions, Dye Deprotonation Levels, and Sensitizing Solvent. ACS Appl. Energy Mater. 2018, 1, 2821-2831.

S3. McCree-Grey, J.; Cole, J. M.; Holt, S. A.; Evans, P. J.; Gong, Y. Dye $\cdots \mathrm{TiO}_{2}$ Interfacial Structure of Dye-Sensitised Solar Cell Working Electrodes Buried under a Solution of $\mathrm{I} / \mathrm{I}_{3}$ Redox Electrolyte. Nanoscale 2017, 9, 11793-11805.

S4. $\quad$ Deng, K.; Cole, J. M.; Rawle, J. L.; Nicklin, C.; Chen, H.; Yanguas-Gil, A.; Elam, J. W.; Stenning, G. B.G. Dye Nanoaggregate Structures in MK-2, N3, and N749 Dye $\cdots \mathrm{TiO}_{2}$ Interfaces That Represent DyeSensitized Solar Cell Working Electrodes. ACS Appl. Energy Mater. 2020, 3, 900-914.

S5. Cooper, C. B.; Beard, E. J.; Vázquez-Mayagoitia, Á.; Stan, L.; Stenning, G. B. G.; Nye, D. W.; Vigil, J. A.; Tomar, T.; Jia, J.; Bodedla, G. B.; Chen, S.; Gallego, L.; Franco, S.; Carella, A.; Justin Thomas, K. R.; Xue, S.; Zhu, X.; Cole, J. M. Design-to-Device Approach Affords Panchromatic Co-Sensitized Solar Cells. Adv. Energy Mater. 2019, 9, 1802820.

S6. $\quad$ Deng, K.; Cole, J. M.; Cooper, J. F. K.; Webster, J. R. P.; Haynes, R.; Al Bahri, O. K.; Steinke, N.-J.; Guan, S.; Stan, L.; Zhan, X.; Zhu, T.; Nye, D. W.; Stenning, G. B. G. Electrolyte/Dye/TiO 2 Interfacial Structures of Dye-Sensitized Solar Cells Revealed by In Situ Neutron Reflectometry with Contrast Matching. Langmuir 2021, 37, 1970-1982.

S7. McCree-Grey, J. Molecular Orientation Dynamics of Dyes in DyeSensitized Solar Cells. Ph.D. Dissertation, University of Cambridge, 2016. 